\title{
Patient Perspectives on Key Symptoms and Preferences for Follow-up After Upper Gastro- intestinal Cancer Surgery
}

\section{Phillip H Pucher}

Guy's and St Thomas' Hospitals NHS Trust: Guy's and St Thomas' NHS Foundation Trust Annie Coombes

Guy's and St Thomas' Hospitals NHS Trust: Guy's and St Thomas' NHS Foundation Trust

Orla Evans

Guy's and St Thomas' Hospitals NHS Trust: Guy's and St Thomas' NHS Foundation Trust Joanna Taylor

Guy's and St Thomas' Hospitals NHS Trust: Guy's and St Thomas' NHS Foundation Trust Jonathan L Moore

Guy's and St Thomas' Hospitals NHS Trust: Guy's and St Thomas' NHS Foundation Trust https://orcid.org/0000-0002-9124-8006

\section{Annabelle White}

Guy's and St Thomas' Hospitals NHS Trust: Guy's and St Thomas' NHS Foundation Trust

\section{Jesper Lagergren}

Karolinska Institute: Karolinska Institutet

\section{Cara Baker}

Guy's and St Thomas' Hospitals NHS Trust: Guy's and St Thomas' NHS Foundation Trust Mark Kelly

Guy's and St Thomas' Hospitals NHS Trust: Guy's and St Thomas' NHS Foundation Trust James A Gossage

Guy's and St Thomas' Hospitals NHS Trust: Guy's and St Thomas' NHS Foundation Trust Jason Dunn

Guy's and St Thomas' Hospitals NHS Trust: Guy's and St Thomas' NHS Foundation Trust

\section{Sebastian Zeki}

Guy's and St Thomas' Hospitals NHS Trust: Guy's and St Thomas' NHS Foundation Trust

\section{Ben Byrne}

University Hospitals Bristol and Weston NHS Foundation Trust

\section{Jervoise Andreyev}

United Lincolnshire Hospitals NHS Trust

Andrew R Davies ( $\square$ andrew.davies1@gstt.nhs.uk)

Guy's and St Thomas' Hospitals NHS Trust: Guy's and St Thomas' NHS Foundation Trust 


\section{Research Article}

Keywords: Survivorship, Symptoms, Esophageal neoplasms, Gastric neoplasms, Esophagectomy, Gastrectomy

Posted Date: November 10th, 2021

DOI: https://doi.org/10.21203/rs.3.rs-1054245/v1

License: (c) (i) This work is licensed under a Creative Commons Attribution 4.0 International License. Read Full License

Version of Record: A version of this preprint was published at Supportive Care in Cancer on March 11th, 2022. See the published version at https://doi.org/10.1007/s00520-022-06922-w. 


\section{Abstract}

Purpose: Long-lasting symptoms and reductions in quality of life are common after oesophago-gastric surgery. Post-operative follow-up has traditionally focussed on tumour recurrence and survival, but there is a growing need to also identify and treat functional sequelae to improve patients' recovery.

Methods: An electronic survey was circulated via a British national charity for patients undergoing oesophago-gastric surgery and their families. Patients were asked about post-operative symptoms they deemed important to their quality of life, as well as satisfaction and preferences for post-operative followup. Differences between satisfied and dissatisfied patients with reference to follow-up were assessed.

Results: Among 362 respondents with a median follow-up of 58 months since surgery (range 3-412), 36 different symptoms were reported as being important to recovery and quality of life after surgery, with a median 13 symptoms per patient. Most (84\%) respondents indicated satisfaction with follow-up. Unsatisfied patients were more likely to have received shorter follow-up than 5 years (27\% among unsatisfied patient vs. $60 \%$ among satisfied patients, $p<0.001)$ and were less likely to have seen a dietitian as part of routine follow-up ( $37 \%$ vs. $58 \%, p=0.005)$.

Conclusion: This patient survey highlights preferences regarding follow-up after oesophago-gastrectomy. Longer follow-up and dietician involvement improved patient satisfaction. Patients reported being concerned by a large number of gastrointestinal and non-gastrointestinal symptoms, highlighting the need for multidisciplinary input and a consensus on how to manage the poly-symptomatic patient.

\section{Introduction}

Upper gastro-intestinal cancer resection (oesophagectomy or gastrectomy) represents a major surgical insult that has a major impact on patients' quality of life [1]. Up to 70\% of patients experience long-term symptoms after surgery [2,3]. Historically, survival rates after surgery for oesophago-gastric cancer were poor, with limited treatment options in the case of disease recurrence. As a result, follow-up practices have focused on survival and the management of tumour recurrence [4, 5]. However, with survival improving, there is a growing need to identify and treat the functional sequelae of oesophago-gastric surgery in order to improve patients' quality of life [6,7]. Additionally, with on-going developments in oncological therapies, the appropriate frequency and duration of follow-up to monitor for disease recurrence remains unknown [8].

There can be little doubt that some patients experience lasting symptoms after oesophago-gastrectomy [9]. Evidence regarding how best to diagnose the underlying cause(s) for these symptoms and how to deliver effective treatment is limited. It is not widely understood that these patients frequently experience multiple symptoms simultaneously which may be caused by a number of co-existing underlying conditions that even experts struggle to predict accurately without diagnostic testing [10]. In the absence of a systematic approach, delays in timely and effective treatment are inevitable and this has a negative 
effect on quality of life after surgery. It may also result in a poor allocation of resources with repeated clinic reviews and inefficient diagnostic testing.

The RESTORE study (REsolution of SympToms after Oesophago-gastric REsection) aims to establish a consensus on the definition, investigation and management of the symptoms and conditions frequently encountered after upper gastro-intestinal cancer resection. As part of this study, a patient questionnaire was circulated via a patient representative body seeking to assess patients' experiences, satisfaction and preferences regarding key issues relating to their follow-up after oesophago-gastric surgery. There was a particular focus on symptoms perceived to be important by patients which might inform the subsequent aspects of the study.

\section{Methods}

An electronic survey was circulated via the website of the Oesophageal Patients Association, a British national charity for patients undergoing oesophago-gastric surgery and their families. Patients who had undergone such surgery were invited to respond to the survey (provided in Supplementary Figure 1). The survey was made available online for a three-week period in March 2021. Survey questions included the year and place of the oesophago-gastric surgery and any additional oncological treatment received. Patients were asked how long they had been, or were due to be, followed up for, what type of specialist performed the majority of follow-up, and whether or not follow-up routinely included review with a dietitian beyond the first post-operative appointment. They were asked how satisfied they felt with their follow-up on a Likert scale of 1-10, and to indicate their preferences for follow-up duration and specialists. Regarding post-operative symptoms, patients were asked to indicate which symptoms they felt were important to overall recovery and quality of life after oesophago-gastrectomy. These were generated from a list of common symptoms as listed in a number of validated questionnaires used in cancer patients, and also included free-text fields for patients to add symptoms not already listed [11].

Following local institutional ethics approval, data were retrieved, anonymised and collated.

Results were presented using descriptive statistics. The intention was to compare patients who reported positive experiences with follow-up, with the remainder of respondents. Results were dichotomised into satisfied (categorised into Likert scale score 7-10 out of 10) or not satisfied (combining dissatisfied (1-3) and intermediately satisfied (4-6)) groups. These groups were compared for follow-up practices and symptoms using Chi-square or Mann-Whitney $U$ tests. Responses were also grouped and compared to identify patients who wished for follow-up to be shorter, the same, or longer than they had experienced, and those who wished to see a specific specialist as part of their follow-up but did not. There was concern that patients who had undergone surgery within less than 5 years may have indicated follow-up duration responses based on care received so far rather than the clinical care team's planned duration of follow-up. A secondary analysis was conducted excluding patients within 5 years of surgery; this did not meaningfully change the results or study conclusion and as such full data are presented here. A p value 
of $<0.05$ was considered significant. Analysis was performed in IBM SPSS statistics (IBM Corp, IBM SPSS statistics Version 27.0. Armonk, NY: USA).

\section{Results}

\section{Patients and treatment}

A total of 362 responses were received, with a median follow-up time of 58 months since surgery (range 3-412 months). Surgical resection was conducted in 71 different hospitals (which included current UK cancer surgery hospitals, private hospitals, and other public hospitals prior to the centralisation of upper gastro-intestinal cancer services in the UK). Perioperative treatments included chemotherapy in 206/362 (57\%), radiotherapy in $5 / 362(1 \%)$, and chemoradiotherapy in $49 / 362(14 \%)$ of cases. Most patients (302/358, 84\%) expressed that they were satisfied with follow-up (Likert score 7-10).

\section{Follow-up practices}

Patient follow-up was variable but was most commonly performed for 5 years $(145 / 352,41 \%)$ after treatment, and by a surgeon (197/358, 55\%) (Table 1). Post-operative follow-up of only 1 year or less was reported by $94 / 352$ (26\%) of patients. Slightly more than half $(198 / 362,55 \%)$ of patients reported routine dietitian review beyond the first post-operative appointment. 
Table 1

Patient reported follow-up practices after oesophago-gastrectomy

\begin{tabular}{|lll|}
\hline & Number & $\%$ \\
\hline Follow-up duration & & \\
\hline$>5$ years & 48 & $14 \%$ \\
\hline 5 years & 145 & $41 \%$ \\
\hline 2 years & 67 & $19 \%$ \\
\hline 1 year & 46 & $13 \%$ \\
\hline 6 months & 28 & $8 \%$ \\
\hline 1 month & 18 & $5 \%$ \\
\hline Primary follow-up speciality & & \\
\hline Surgeon & 197 & $55 \%$ \\
\hline Dietitian / nurse & 97 & $27 \%$ \\
\hline Oncologist & 51 & $14 \%$ \\
\hline Gastroenterologist & 13 & $4 \%$ \\
\hline Routine dietitian review & & \\
\hline Yes & 198 & $55 \%$ \\
\hline No & 164 & $45 \%$ \\
\hline Satisfaction with follow-up & & \\
\hline Positive (Likert 7-10) & 302 & $84 \%$ \\
\hline Neutral (4-6) & 37 & $10 \%$ \\
\hline Negative (1-3) & 19 & $5 \%$ \\
\hline
\end{tabular}

\section{Patient preferences in follow-up}

The majority of patients $(275 / 356,77 \%)$ expressed a preference for follow-up of 5 years or more (Table 2). Comparing follow-up duration received to reported patient preferences, half of the patients $(177 / 362$, $49 \%$ ) felt the follow-up duration they had received was appropriate, whereas 152/362 (42\%) wished follow-up was longer. There was no relationship between median time elapsed since surgery and satisfaction. Few patients (33/362, 9\%) expressed a desire for shorter follow-up. 
Table 2

Patient reported follow-up preferences after oesophago-gastrectomy

\begin{tabular}{|lll|}
\hline & $\mathbf{n}$ & $\%$ \\
\hline Preferred follow-up duration & & \\
\hline Longer than 5 years & 103 & $29 \%$ \\
\hline 5 years & 172 & $48 \%$ \\
\hline 2 years & 33 & $9 \%$ \\
\hline 12 months & 18 & $5 \%$ \\
\hline 6 months & 10 & $3 \%$ \\
\hline 1 month & 20 & $6 \%$ \\
\hline Preferred follow-up speciality & & \\
\hline Surgeon & 210 & $57 \%$ \\
\hline Dietitian / nurse & 90 & $23 \%$ \\
\hline Oncologist & 51 & $14 \%$ \\
\hline
\end{tabular}

\section{Factors affecting satisfaction with follow-up}

There was no difference between groups that were unsatisfied and satisfied with the follow-up regarding the time elapsed since treatment or the number of symptoms reported as being important (Table 3 ). Unsatisfied patients were more likely to have received shorter follow-up than 5 years $(27 \%$ among unsatisfied patient vs. $60 \%$ among satisfied patients, $p<0.001$ ) and identified follow-up duration as a reason for dissatisfaction (30\% of unsatisfied patient were satisfied with their follow-up duration vs. $53 \%$ of satisfied patients, $p=0.002$ ). There was no difference between groups for which specialists performed the follow-up, but there was a greater proportion of unsatisfied patients who expressed a preference for follow-up to include a surgeon, whose follow-up was led by a non-surgeon ( $28 \%$ vs. $9 \%, p<0.001)$. Unsatisfied patients were less likely to have seen a dietitian as part of routine follow-up ( $37 \%$ vs. $58 \%$, $p=0.005)$. 
Table 3

Comparison of patient groups satisfied vs. not satisfied with follow-up after oesophago-gastrectomy

\begin{tabular}{|c|c|c|c|}
\hline & Unsatisfied & Satisfied & p-value \\
\hline Months since treatment (months) & $50(4-412)$ & $60(3-404)$ & $0.498^{*}$ \\
\hline Number of reported important symptoms & $12(0-31)$ & $13(0-33)$ & $0.818^{*}$ \\
\hline \multicolumn{4}{|l|}{ Duration follow-up received } \\
\hline 1 month & $8 / 55(15 \%)$ & $10 / 293(3 \%)$ & \multirow{6}{*}{$<0.001^{* *}$} \\
\hline 6 months & $9 / 55(16 \%)$ & $18 / 293(6 \%)$ & \\
\hline 1 year & $14 / 55(25 \%)$ & $30 / 293(9 \%)$ & \\
\hline 2 years & $9 / 55(16 \%)$ & $58 / 293(20 \%)$ & \\
\hline 5 years & $8 / 55(15 \%)$ & $136 / 293(46 \%)$ & \\
\hline$>5$ years & $7 / 55(13 \%)$ & $41 / 293(14 \%)$ & \\
\hline Desired follow-up duration received & $17 / 56(30 \%)$ & $160 / 302(53 \%)$ & $0.002^{* *}$ \\
\hline \multicolumn{4}{|l|}{ Follow-up primarily performed by } \\
\hline Surgeon & $30 / 53$ & $165 / 301$ & \multirow{4}{*}{$0.892^{\star *}$} \\
\hline Nurse/dietitian & $14 / 53$ & $82 / 301$ & \\
\hline Oncologist & $8 / 53$ & $42 / 301$ & \\
\hline Gastroenterologist & $1 / 53$ & $12 / 301$ & \\
\hline Desired but did not see surgeon & $16 / 56(28 \%)$ & $27 / 302(9 \%)$ & $<0.001^{* *}$ \\
\hline Desired but did not see oncologist & $0 / 56$ & $0 / 302$ & $1.000 * *$ \\
\hline Desired but did not see nurse or dietitian & $4 / 56(7 \%)$ & $32 / 302(10 \%)$ & $0.430 * *$ \\
\hline Dietitian was seen as part of routine follow-up & $21 / 56(37 \%)$ & $175 / 302(58 \%)$ & $0.005^{\star *}$ \\
\hline
\end{tabular}

\section{Symptoms after oesophago-gastrectomy}

In all, 36 different symptoms (comprising 31 pre-defined symptoms listed in the questionnaire and 5 additional symptoms added via free-text function by respondents) were reported by patients as being important to recovery and quality of life after oesophago-gastrectomy (Table 4). Some of these included overlapping symptom complexes (e.g. dumping syndrome and dizziness after meals). Symptoms of concern were shared by a large proportion of patients, with all 31 pre-defined symptoms identified by 
more than $20 \%$ of respondents. Patients reported a median of 13 (range $0-33$ ) of symptoms they deemed important to their quality of life after surgery. These included both gastrointestinal and nongastrointestinal symptoms. The most frequent symptoms reported were heartburn $(293 / 362,81 \%)$ and early satiety $(269 / 362,74 \%)$. 
Table 4

Patient-reported important symptoms after oesophago-gastrectomy

\begin{tabular}{|c|c|c|}
\hline Symptom & $\mathbf{n}$ & $\%$ \\
\hline Heartburn or acid regurgitation & 293 & 80.9 \\
\hline Feeling full after small amount of food & 269 & 74.3 \\
\hline Need to rush to open bowels & 252 & 69.6 \\
\hline Tiredness / lethargy & 251 & 69.3 \\
\hline Difficulty swallowing solids & 248 & 68.5 \\
\hline Weight loss & 237 & 65.5 \\
\hline Abdominal cramps / trapped wind & 231 & 63.8 \\
\hline Reduced appetite & 209 & 57.7 \\
\hline Nausea / feeling sick & 209 & 57.7 \\
\hline Belching or burping & 206 & 56.9 \\
\hline Bowel frequency / consistency & 198 & 54.7 \\
\hline Upper abdominal pain/ discomfort & 195 & 53.9 \\
\hline Dizziness / light headed after meals & 193 & 53.3 \\
\hline Vomiting / being sick/ retching & 184 & 50.8 \\
\hline Lower abdominal pain/ discomfort & 181 & 50.0 \\
\hline Stomach / abdominal gurgling & 175 & 48.3 \\
\hline Difficulty swallowing liquids & 158 & 43.6 \\
\hline Leakage / soiling or lack of control of the bowel & 157 & 43.4 \\
\hline Excessive passing of wind from your bottom & 156 & 43.1 \\
\hline Abdominal bloating / distension & 147 & 40.6 \\
\hline Experienced change in taste & 146 & 40.3 \\
\hline Feeling that you have not emptied your bowel properly & 139 & 38.4 \\
\hline Greasy, pale or oily stool & 138 & 38.1 \\
\hline Woken from sleep to have bowels open & 100 & 27.6 \\
\hline Bleeding from your bottom & 93 & 25.7 \\
\hline Hiccups & 84 & 23.2 \\
\hline
\end{tabular}




\begin{tabular}{|lll|}
\hline Symptom & $\mathbf{n}$ & $\%$ \\
\hline Mucus in the stool & 84 & 23.2 \\
\hline Experienced change in smell & 79 & 21.8 \\
\hline Bad breath / halitosis & 77 & 21.3 \\
\hline Itchiness around the bottom & 77 & 21.3 \\
\hline Pain around your bottom & 75 & 20.7 \\
\hline Dumping* & 54 & 14.9 \\
\hline Reflux* & 29 & 8.0 \\
\hline Thoracotomy / rib pain* & 17 & 4.7 \\
\hline Sleep disturbance* & 14 & 3.9 \\
\hline Psychological distress* & 11 & 3.0 \\
\hline *Added manually via free-text fields by respondents & & \\
\hline
\end{tabular}

\section{Discussion}

This is the first study, to our knowledge, to examine patient preferences and satisfaction with follow-up after oesophago-gastrectomy for cancer. This survey highlights the variability in practice in relation to follow-up duration and the healthcare professionals delivering it. Patients identified a large number of symptoms which they considered important to address as part of the follow-up process. Those reporting lower satisfaction scores received shorter follow-up, and were less likely to have seen a surgeon or had regular input from a dietitian.

There is a paucity of evidence in relation to optimal follow-up after oesophago-gastric cancer surgery. One study previously highlighted that follow-up arrangements after cancer treatment in general, which usually involve outpatient appointments at cancer centres, do not meet all cancer survivors' needs and provide questionable value for money [12]. They highlighted a need to transform cancer care from a 'onesize fits all' approach to one based on the assessment of individual needs and preferences. The report of the Independent Cancer Taskforce identified that a large proportion of current cancer costs within the National Health Service (NHS) in the United Kingdom relate to treating people who are in the survivorship phase and that more tailored care has the potential to reduce costs through reducing tumour recurrences, better management of side-effects and supporting people to live well [13].

The large number of both gastrointestinal and non-gastrointestinal symptoms reported by patients as 'important' after oesophago-gastrectomy is in agreement with the recent LASER study, in which $67 \%$ of responding patients reported troublesome symptoms at a median of 4.3 years after oesophagectomy [2]. All of the symptoms listed in the present study were felt to be important by $20 \%$ or more of participants, 
thus justifying their inclusion in future studies assessing the symptom burden in this patient group. Given the overlap of symptoms that may be attributed to the varying conditions that commonly affect patients after oesophago-gastrectomy, it remains to be seen whether symptom combinations may be used to predict the underlying cause(s) or whether systematic investigations are required. Either way, this survey forms a patient-led baseline from which a standardised approach to the management of post-operative symptoms may be considered. The proportion of patients reporting concerns over potential mental health symptoms such as sleep disturbance or psychological distress was low and may reflect under-reporting of these issues [3].

Whilst the majority of patients were satisfied with their care overall, this study has demonstrated important differences between this group and the remaining unsatisfied patients. These differences highlight areas which centres might consider when seeking to improve post-treatment follow-up protocols. Patient satisfaction was not associated with time elapsed since treatment, suggesting the risk of recall bias, or satisfaction being related to temporal trends in practice, was low. There were also no differences in the number of symptoms highlighted by satisfied and unsatisfied patients.

The majority of patients expressed a desire to be seen by a surgeon as part of their post-operative care. Understandably, patients feel a strong affiliation to the surgeon who performed their operation, despite the fact that many aspects of symptom management fall outside traditional surgical expertise. Unsurprisingly, dietitian involvement in routine follow-up was higher in patients who reported high satisfaction scores although this did not align with the preferred specialisms involved in follow-up as specified by patients. The reasons for this discordance are unclear. One aspect may relate to the survey design, which did not include descriptions of the roles of various specialties, meaning patients based their responses on their personal experiences alone. Socioeconomic, cultural, and educational patient factors have also been shown to play a role in preferences for post-operative follow-up [14]. The importance of dietitian support throughout the surgical pathway is crucial for oesophago-gastrectomy patients, who are at high risk of malnutrition and gastrointestinal complications $[15,16]$. Patient understanding of these factors may underlie the preferences reported here to a degree. The fact that only $55 \%$ of patients reported routine involvement of a dietitian stands in stark contrast, for example, to a recent Australian and New Zealand survey in which surgeons reported always involving dietetic support postoperatively [16]. Given that the majority of symptoms reported by patients in this study were gastrointestinal in nature, it would seem imperative to increase the rate of dietitian support to manage common symptoms such as reflux or dumping as well as malnutrition. Access to gastroenterology expertise and the means to investigate for the underlying conditions that often cause the symptom burden would also be important in the design of any follow-up model.

Patients expressed a preference for longer follow-up. While current practices largely support follow-up for 5 years after surgery $[5,18]$, there was support ( $29 \%$ of respondents) for follow-up to continue beyond this, although the preferred duration beyond 5 years was not elucidated in the survey. This reflects the general desire for longer contact with healthcare professionals after complex procedures, as demonstrated in other studies [14, 19-20]. Other more complex issues such as follow-up "as required", 
rather than regular pre-set appointments, or future moves towards online symptom reporting triggering follow-up were beyond the remit of this survey, but these issues certainly merit future consideration.

Some methodological limitations of this study deserve discussion. This cross-sectional survey was not fully representative of all oesophago-gastrectomy patients as it excluded those who had not survived or did not take part in the questionnaire. By enrolling patients who self-selected for inclusion via a national patient support group, potential selection bias was introduced. The design of the survey asked patients about their own experiences; responses may have been different if a scenario-based questionnaire was used or if the relative merits of follow-up by different specialists as part of different models were explained as part of the survey. Some questions were asked in an exclusive manner which may not have captured the full scope of the follow-up; the fact that few patients reported that their follow-up care was primarily carried out by a gastroenterologist, for example, must be differentiated from patients where gastroenterologists were additionally consulted for the on-going management of their symptoms. This may not have been fully captured by the survey.

Follow-up after major cancer surgery has multiple aims including tumour recurrence surveillance, symptom management and patient reassurance. The former was beyond the scope of this study but is clearly important, given recent advances in second and third-line oncological therapies. The overall goal is to restore quality of life after cancer treatment. While this study aimed to characterise the symptoms patients' deemed important, and their overall satisfaction with follow-up, more work is needed to specifically identify what patients want and benefit from post-operatively and how this aligns with the medical evidence-base and the resources realistically available. Further research is crucial to devising a follow-up regimen which optimises both clinical and patient-reported outcomes after oesophageal surgery.

In conclusion, this large patient survey highlights the important preferences of patients regarding followup after surgery for oesophageal or gastric cancer. Routine dietitian involvement was only reported by half of patients yet was associated with greater patient satisfaction with the follow-up received. Patients were concerned by a large number of gastrointestinal and non-gastrointestinal symptoms, highlighting the need for multidisciplinary input and a consensus on how to best investigate and manage the polysymptomatic patient.

\section{Declarations}

Declarations: The authors declare no conflict of interest

Funding: There were no sources of funding for this study

\section{References}

1. Kauppila JH, Johar A, Lagergren P. Medical and Surgical Complications and Health-related Quality of Life After Esophageal Cancer Surgery. Ann Surg 2020; 271(3):502-508. 
2. Markar SR, Zaninotto G, Castoro C, et al. Lasting Symptoms After Esophageal Resection (LASER): European Multicenter Cross-sectional Study. Ann Surg 2020.

3. Bouras G, Markar SR, Burns EM, et al. The psychological impact of symptoms related to esophagogastric cancer resection presenting in primary care: A national linked database study. Eur $J$ Surg Oncol 2017; 43(2):454-460.

4. Chew T, Bright T, Price TJ, et al. Follow-Up Practices of Surgeons and Medical Oncologists in Australia and New Zealand Following Resection of Esophagogastric Cancers. Ann Thorac Cardiovasc Surg 2017; 23(5):217-222.

5. Moyes LH, Anderson JE, Forshaw MJ. Proposed follow up programme after curative resection for lower third oesophageal cancer. World J Surg Oncol 2010; 8:75.

6. Anderson LA, Tavilla A, Brenner $\mathrm{H}$, et al. Survival for oesophageal, stomach and small intestine cancers in Europe 1999-2007: Results from EUROCARE-5. Eur J Cancer 2015; 51(15):2144-2157.

7. Andreyev HJN, Muls AC, Shaw C, et al. Guide to managing persistent upper gastrointestinal symptoms during and after treatment for cancer. Frontline Gastroenterol 2017; 8(4):295-323.

8. Abate E, DeMeester SR, Zehetner J, et al. Recurrence after esophagectomy for adenocarcinoma: defining optimal follow-up intervals and testing. J Am Coll Surg 2010; 210(4):428-35.

9. Grace EM, Shaw C, Lalji A, et al. Nutritional status, the development and persistence of malnutrition and dietary intake in oesophago-gastric cancer: a longitudinal cohort study. J Hum Nutr Diet. 2018 Dec;31(6):785-92.

10. Muls AC, Klimova K, Andreyev HJN. Clinical decision-making in managing changes in gastrointestinal function following cancer therapies: Is experience enough? Eur J Cancer Care (Engl). 2018 Jan;27(1).

11. Lagergren $P$, Fayers $P$, Conroy $T$, et al. Clinical and psychometric validation of a questionnaire module, the EORTC QLQ-025, to assess health-related quality of life in patients with cancer of the oesophagus, the oesophagogastric junction and the stomach. Eur J Cancer. 2007;43:2066-2073.

12. Richards $M$, Corner $J$ and Maher J. The National Cancer Survivorship Initiative: new and emerging evidence on the ongoing needs of cancer survivors. British Journal of Cancer 2011; 105, S1 - S4.

13. Report of the Independent Cancer Taskforce (2015). Achieving World-Class Cancer Outcomes A Strategy for England 2015-2020. Available at:

https://www.cancerresearchuk.org/sites/default/files/achieving_world-class_cancer_outcomes__a_strategy_for_england_2015-2020.pdf

14. Damery S, Biswas $M$, Billingham $L$, et al. Patient preferences for clinical follow-up after primary treatment for soft tissue sarcoma: a cross-sectional survey and discrete choice experiment. Eur $\mathrm{J}$ Surg Oncol 2014; 40(12):1655-61.

15. Ligthart-Melis GC, Weijs PJ, te Boveldt ND, et al. Dietician-delivered intensive nutritional support is associated with a decrease in severe postoperative complications after surgery in patients with esophageal cancer. Dis Esophagus 2013; 26(6):587-93. 
16. Huddy JR, Macharg FM, Lawn AM, et al. Exocrine pancreatic insufficiency following esophagectomy. Dis Esophagus 2013; 26(6):594-7.

17. Lahoud J, Bazzi K, Yeo D, et al. Survey of nutritional practices in total gastrectomy and oesophagectomy procedures. Nutr Diet 2019; 76(2):135-140.

18. Allum WH, Blazeby JM, Griffin SM, et al. Guidelines for the management of oesophageal and gastric cancer. Gut 2011; 60(11):1449-72.

19. Blom RL, Nieuwkerk PT, van Heijl M, et al. Patient preferences in screening for recurrent disease after potentially curative esophagectomy. Dig Surg 2012; 29(3):206-12.

20. McFerran E, Boeri M, Kee F. Patient Preferences in Surveillance: Findings From a Discrete Choice Experiment in the "My Follow-Up" Study. Value Health 2020; 23(10):1373-1383.

\section{Supplementary Files}

This is a list of supplementary files associated with this preprint. Click to download.

- Supplementaryfigure1.pdf 